\title{
Creating a subcorpus of a heritage language on the example of Yiddish
}

\author{
Valentina Fedchenko ${ }^{1}$, Ilia Uchitel $^{2}$ \\ ${ }^{1}$ Department of Jewish Culture, St. Petersburg State University, Russia \\ ${ }^{2}$ School of linguistics, Higher School of Economics in Moscow, Russia \\ https://doi.org/10.36505/ExLing-2016/07/0012/000271
}

\begin{abstract}
The paper presents a Yiddish heritage subcorpus on the basis of the Corpus of Modern Yiddish. The contemporary status of the Yiddish language and the absence of monolingual speakers nowadays makes it perfect candidate for research within the framework of heritage languages. Yiddish exists in different sociolinguistic contexts and forms plenty of bilingual pairs. Corpus-linguistic approach, especially in corpora with multimedia utilities and L2 component, enlarges the variety of possible instruments and subjects of research. The paper discusses practical issues of creating and using a multimodal corpus of the Yiddish language with a special focus on the more recently added subcorpus of recorded interviews with L2 speakers of Yiddish, while analyzing the corpus architecture, the corpus representativity, L2 corpus marking.

Key words: corpus linguistics, Yiddish, multimedia corpus, L2 corpus
\end{abstract}

\section{Yiddish as a heritage language and L2}

This short article presents the project of developing corpora tools aimed at producing quantitative research of Yiddish as a heritage and learned as second language (L2).

Yiddish language is a West-Germanic language spoken mainly by Ashkenazi Jews. In the beginning of XXth century it was main language of communication, both oral and written, for Jews in central and eastern Europe, including vast lands of former Russian and Austro-Hungarian Empires. Since then the dialectal diversity in Yiddish remains very high. After the Holocaust and mass migration to Israel and the US, Yiddish speakers has nearly disappeared in their original language areas (i.e. today Poland and Ukraine). Still, language situation in different areas, vary. While in Poland and Lithuania Yiddish ceased to be language of communication shortly after the WW2, in Ukraine, Belarus and Moldova, where the number of Jewish survivors was much bigger, Yiddish continued to be used and even be acquired by post-war children till the 1980s - the beginning of mass migration to Israel and other countries.

Having lost a great number of its speakers, the Yiddish language is still spoken by some communities in Israel and the US. It is still

ExLing 2016: Proceedings of $7^{\text {th }}$ Tutorial and Research Workshop on Experimental Linguistics, 27 June - 2 July 2016, Saint Petersburg, Russia 
considered to be an important part of Jewish identity, with some scholars qualifying it as a language which is in some cases used as "postvernacular" [Avineri 2012: 25] and surpasses the ordinary communicative use by developing extra-functions (i.e. cultural, symbolical). As an example of such use we can mention widespread use of Yiddish in several language programs, such as "Yidish Vokh" ('Yiddish Week') or "Yidish dorf" ('Yiddish Village'), where participants (numerously not native speakers) are supposed to speak only Yiddish. This "postvernacular" use continues in the Internet with heritage activists which use Yiddish, for example, in their everyday Facebook activities.

In the past decade, the subject of heritage Yiddish use (mainly in the US) have been studied by several scholars in such works as [Shandler 2008], [Avineri 2012], [Sadan 2011], [Levine 2000]. In addition to these thorough sociolinguistic descriptions of the current practices, there are small amount of works focusing on analysis of specific features of Yiddish as a heritage language. We can note, for example, [Safadi 2000], discussing noun gender and case differences between groups of heritage and native speakers, and, partially, [Levine 2000] with discussion on choice of auxiliary in perfect tense among heritage and non-heritage speakers.

\section{Corpus data}

One of the most valuable projects made during recent years are Corpus of Modern Yiddish (http://web-corpora.net/YNC/search/) and Yiddish Multimedia Corpus (http://webcorpora.net/YiddishMultimediaCorpus/search/). First one includes documents representing language of press and fiction of the XIXth till late XX centuries, including modern documents. Second corpus presents annotated audio records of authentic Yiddish speech, with speakers coming from various dialect areas. It includes 10 files: lectures and field recordings. With online search available, these sources give a great possibility for performing quantitative studies of Yiddish language, as well as learning Yiddish as second language.

CMY contains currently 4150933 tokens from 3662 documents. The largest part of it is press with a share of $78.43 \%$ is mostly represented by archive of "Forverts" newspaper, publishing in the US, with issues dating from 2004. Some of the press text authors are not native speakers, or, to some extent, are heritage speakers.

Therefore, the first step for construction of heritage and L2 subcorpus is looking for sociolinguistic characteristics of the authors. Such characteristics should include, at least, type of language knowledge 
(native/heritage/second), first language (if appreciable), year of birth of the speaker. Some additional information, however, would enrich the set: for example, details about parents' place of birth and their knowledge of Yiddish.

Though there are many parsed documents of this type in CMY and there can be some findings results just after adding sociolinguistic information, the overall result cannot be considered as fully reliable and representative for several reasons: 1 . texts published in press are preedited, even if in some of them (i.e. ultraorthodox Hasidic newspapers) some idiolect features are tolerated; 2 . print text genre represent different linguistic peculiarities, comparing with colloquial language examples. Yiddish multimedia corpus either cannot provide data from heritage and L2 speakers.

In order to collect relevant information, oral interviews with heritage speakers can be produced. While the advantages of this approach are clear, there are some serious shortcomings. The most important problem is the time-consuming manual transcription of interviews. This can be avoided by interviewing consultants in writing, primarily, using Internet. However, in the written data we can hardly find some traces (i.e spelling mistakes) to phonological features of consultant's speech. In addition, such interviews can be conducted with a limited number of speakers, who use internet for some communication in Yiddish, with arising problem of transcription processing. Moreover, there is a possibility to proceed automatically some amount of independently produced texts by a limited set of "language activists", who use Yiddish in their public communication on Internet (basically, in communication on Facebook). This material which demonstrates a real language use, can be very useful.

\section{Text processing and access}

A well-developed tagging engine already exists for Corpus of Modern Yiddish, so that the inserted texts can be quickly morphologically parsed (with certain inaccuracy due to homonymy). The heritage subcorpus can be built into the CMY, with adding search by sociolinguistic metadata, or it can be hosted independently, but will share the CMY platform.

One of the greatest difficulties in building a L2 subcorpus is the marking of mistakes or "non-standard language features". It takes remarkably much time to find and classify a mistake, which can occur at all levels of language. Even for Russian Learning Corpus team of native Russian speakers a special error-checking engine was necessary for facilitating the mistake marking. 
As the stage of gathering data for corpus is not fulfilled yet, the timeconsuming process of mistake marking should be postponed. Nevertheless, the option to search by mistakes (or "distinct features") is very important not only for theoretical reasons, but for applied purposes (as language teaching) as well.

However, when applying this principle to Yiddish data, we can face a different kind of problem. The error tagging is quite difficult even for native speakers. A vague standard language and a high dialectal diversity makes this task nearly impossible. Probably, another way to highlight distinct features of heritage Yiddish should be used. One simple way to do it is to use bi-grams and tri-grams of some lemmas and then compare results of heritage and non-heritage language. This method was used in automatic error-detection tool made for Russian Learner Corpus [Klyachko et al. 2013]. A spell checking engine would be very useful for tracing mistakes in certain documents. There are some Yiddish spell checkers, elaborated by YIVO.

One more problem concerns the analysis of phonological features in the heritage speech. In several corpus projects (i.e. YMC) the original speech was transcribed according to standard Yiddish rather than phonetically. That leads to difficulties in quantitative analysis of phonology. Unfortunately, transcribing audio according to IPA alphabet would take tremendous efforts and can't be done at the time. However, YMC interface allows to look for individual word and then find it easily in the audio file. Therefore, inserting new heritage records into the YMC engine and tagging it seems to be the best solution at time.

\section{Acknowledgements}

The authors thank for financial support the Russian Science Foundation (project 15-1800062, St. Petersburg State University).

\section{References}

Avineri, N.R. 2012. Heritage language socialization practices in secular Yiddish educational contexts. Disertation in Applied Linguistics, University of California.

Klyachko E., Arkhangelskiy T., Kisselev O., Rakhilina E. 2013. Automatic error detection in Russian learner language. Corpus Linguistics. Lancaster, UK.

Levine, G.S. 2000. Incomplete L1 acquisition in the immigrant situation. Tübingen, Niemeyer.

Sadan, T., 2011. Yiddish on the Internet. Language and Communication, 31(2), 99-106.

Safadi, M. 2000. Yiddish: its survival in an English-dominant environment. Dissertation, University of California.

Shandler, J. 2008. What is American Jewish Culture? In Raphael, M. L. (ed.), The Columbia History of Jews and Judaism in America, 337-365. Columbia University Press, New York. 